\title{
Radio Continuum Emission in the Resonance-Ring Galaxies NGC 1433 and NGC 6300
}

\author{
Hemant Shukla and Stuart D. Ryder ${ }^{1}$ \\ Department of Physics $\&$ Astronomy, University of Alabama, Box \\ 870324, Tuscaloosa, AL 35487-0324
}

\section{Introduction}

NGC 1433 is a nearly face-on prototypical ringed, barred galaxy. It has a nuclear ring, an inner ring and an outer pseudoring. Also present are symmetrical spiral arcs, also called "plumes", leading the central bar. Based on a study using multicolor surface photometry and $\mathrm{H} \alpha$ Fabry-Perot interferometry, Buta (1986) was able to associate each of the ring features with a specific orbital resonance. NGC 6300 , having almost an identical Hubble type and distance to that of NGC 1433, provides for an ideal comparison. It has no nuclear or outer rings. However, the inner ring is quite broad and dusty (Buta 1987). Radio continuum data were obtained with the Australia Telescope Compact Array (ATCA) at 1.38 $\mathrm{GHz}$ (bandwidth $=128.0 \mathrm{MHz}$ ) with maximum baselines of $1.5 \mathrm{~km}$, yielding a resolution of $\sim 30^{\prime \prime}$ and r.m.s. noise per map of $0.2 \mathrm{mJy} \mathrm{Beam}^{-1}$ for both of the galaxies. The $\mathrm{H} \alpha+\mathrm{N}[\mathrm{II}]$ images for both of the galaxies were obtained with the CTIO $1.5 \mathrm{~m}$ telescope.

\section{NGC 1433}

Figure 1 is a contour map of radio continuum emission at $1.38 \mathrm{GHz}$ from NGC 1433 superimposed onto its $\mathrm{H} \alpha+\mathrm{N}[\mathrm{II}]$ image. Coincident with the optical position of the Type II SN 1985P (Barbon et al. 1989), marked by the cross towards the northeast side of the inner ring, is a radio source of flux density $0.5 \mathrm{mJy}$. The continuum contours also appear to align well with the HII region complexes on either end of the bar. We find the integrated flux density for the eastern complex to be of the order of $3.4 \mathrm{mJy}$, while that for the western complex is about $3.7 \mathrm{mJy}$. These fluxes are comparable with the flux density of the nuclear source which is $3.4 \mathrm{mJy}$.

\section{NGC 6300}

In contrast NGC 6300 shows a different pattern of continuum emission. Figure 2 is a contour map of radio continuum emission at $1.38 \mathrm{GHz}$ from NGC 6300 superimposed onto its $\mathrm{H} \alpha+\mathrm{N}[\mathrm{II}]$ image. The galaxy is a Type 2 Seyfert and shows an unresolved strong nuclear source. Radio emission traces the inner ring

\footnotetext{
${ }^{1}$ Current Address: School of Physics, University of New South Wales, Sydney 2052, Australia
} 


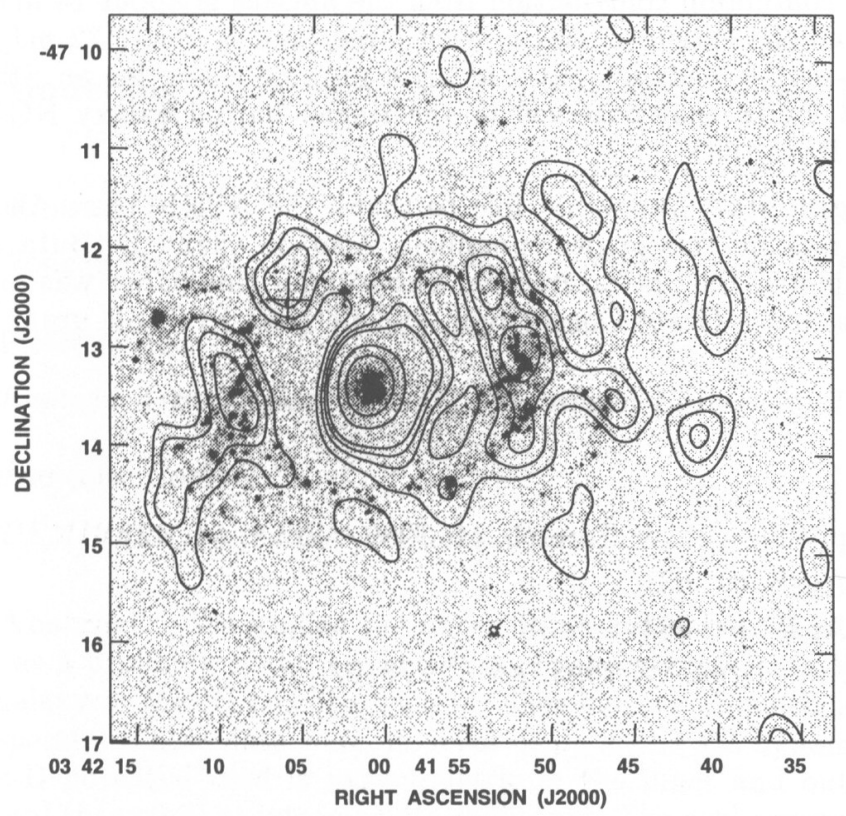

Figure 1. NGC 1433, radio continuum overlaid on $\mathrm{H} \alpha+\mathrm{N}[\mathrm{II}]$ image

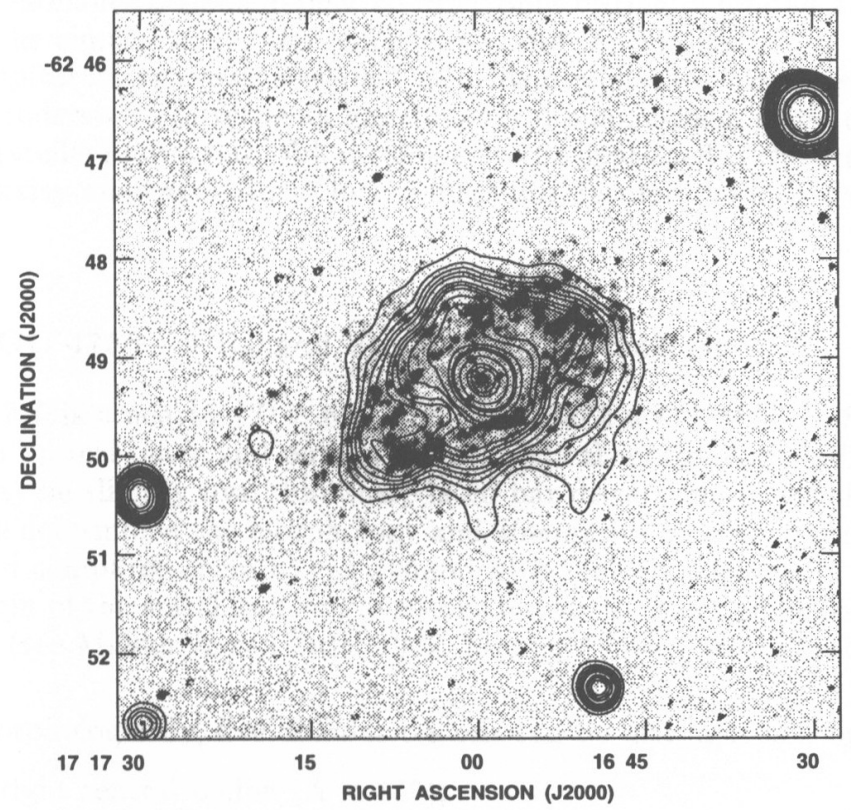

Figure 2. NGC 6300, radio continuum overlaid on $\mathrm{H} \alpha+\mathrm{N}[\mathrm{II}]$ image 
well. The radio continuum contribution from the nucleus is about $14 \mathrm{mJy}$. The contribution from the extended disk is even larger and is about $72 \mathrm{mJy}$. This gives a nucleus-to-disk emission ratio of 0.19 , unusually low for an SBb type galaxy, but not unlike that observed in the ringed barred galaxy NGC 4736 (Mulder \& van Driel 1993).

Acknowledgments. We would like to thank the ATNF Time Allocation Committee for making this project possible. Also we thank R. Buta and D. Crocker for supplying the optical images. Support for this paper was provided by the Alabama Space Grant Consortium, and by NSF EPSCoR grant EHR 9108761 .

\section{References}

Barbon, R., Cappellaro, E., \& Turatto, M. 1989, A\&AS, 81, 421

Buta, R. 1986, ApJS, 61, 631

Buta, R. 1987, ApJS, 64, 383

Mulder, P. S. \& van Driel, W. 1993, A\&A, 272, 63 